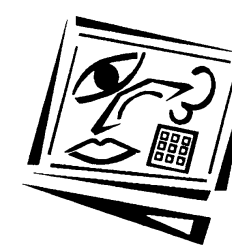

\title{
Towards a new generation of simulation models in public health education
}

\author{
Deon V Canyon \\ James Cook University \\ David N Podger \\ Craftsmen Products Pty Ltd
}

\begin{abstract}
This article sets the scene for the development of an educational simulation software package based on multimedia, decision support systems, risk estimates, project outcomes and evaluation. It begins by looking at the history and current use of educational software and its application to the education of public health professionals. Then, it previews accompanying technological advances that were essential precursors to the facilities now available and reviews learning theories relevant to the development of problem solving simulation models. Possible candidates for providing more effective educational solutions, such as decision support systems, modelling and simulation software are reviewed and the design of a new generation, fully integrated simulation model is discussed.
\end{abstract}

\section{Introduction}

Online education is growing at a fast pace in line with the development of educational technologies. In a short span, since the general availability of the World Wide Web in 1995, significant advances and changes have occurred and the evolution of online education has witnessed a proliferation of software tools that have acted both to facilitate and enhance the educational experience. This transformation in educational technology and processes will inevitably impact on the function of educators. Interested academics are already considering the roles they might be expected to play in the future.

There is an accelerating global need to provide distance education in a form that is both appealing and effective. At the same time, the web technology that can deliver it is growing and developing at a staggering rate. Bright Planet released a study estimating that the World Wide Web 
(Web) is 500 times larger than the segment covered by standard search engines such as Google and Alta Vista. It claimed that the Web now holds in excess of 550 billion documents (Bergman, 2000). Online education is being driven along by these hi-tech advances, but the question: 'How does the provision of educational material over the Web differ from traditional print based material?' still needs to be pondered.

Another factor driving change is the ever diminishing university budget line. The educational system is being challenged to provide quality education with fewer resources (Zenger and Walker, 1999). Yet another factor is the change in the role of teachers and trainers from traditional information givers to learning facilitators. Change is also being driven by the advent of competency based training and the need to deliver self paced instruction (Pellone, 1995).

The area of interest for this paper, educational public health simulation software, is framed within a brief review of the history of public health in Australia and the accompanying technological developments that were essential precursors to the facilities now available.

\section{Public health training}

Public health is acknowledged to have originated from pragmatic reactions to the effects of uncontrollable epidemic and endemic disease outbreaks on community health. An early example of this occurred in Venice where formal quarantine regulations were implemented in the 1300s. Another influencing factor, driven in part by the child health movements of the late 1800s, was the philanthropic wish to provide a minimum standard of well being to socio-economically deprived children (Anon, 2001).

Large catastrophic epidemics associated with urban overcrowding were not manageable by individual medics and required the creation of health departments and health training institutions. Around 1910, the United States of America founded a number of these departments and promoted specialist training for their physicians, nurses and sanitation engineers. Training was initially developed at the Massachusetts Institute of Technology, Harvard University, and Johns Hopkins University (Anon, 2001). The concept of providing a general health education program integrating elements of academic theory and applied or professional knowledge emerged, and formal public health education was born.

The contemporary story of public health education and training in Australia really began in 1985 with the Kerr White Report, which argued for a redistribution of funds from the School of Public Health and Tropical 
Medicine to new public health institutions across Australia. This report had a profound and lasting impact and in 1986/7 the Commonwealth Department of Health established the Public Health Education and Research Program (PHERP) and the Australian Institute of Tropical Medicine was re-established under James Cook University of North Queensland after an absence of 56 years.

But public health education still had a long way to go. In 1988, a report to the Australian Health Ministers' Advisory Council on Continuing Education for Primary Health Care in Australia stated that "continuing education was piecemeal and poorly coordinated with little focus on preventive, multi-professional and intersectoral approaches to health" (NPHP, 1998). Public benefit then became the focus and in 1990, a report on workforce issues for public health by the Public Health Association of Australia described four levels of training: specialists, practitioners, general and associated health workers, and consumer and community organisations. This report drew attention to the interlinked yet scattered work environments of public health professionals. PHERP was revised and refunded in 1995, and actively supported postgraduate public health training programs around the country. Several educational problem areas became apparent such as the need for improved training access and delivery, quality control mechanisms, health promotion priorities and equity in access to training. In 1997 a quality enhancement process was initiated by PHERP to address these issues and many others. The recent formation of the Australian Network of Academic Public Health Institutions (ANAPHI) makes it clear that public health education and training in Australia is still a concern. ANAPHI designed a website in 2001 to facilitate information exchange and to promote education and research dialogue (ANAPHI, 2001).

Advances in flexible public health training are closely linked with technological advances.

\section{Technology and training}

When public health was just finding its feet, the use of technology was restricted to instructional films and television. In the 1950s and beyond, interest in instructional television experienced significant growth, and film rental libraries noted increased interest in individualised instruction (An, 2001). A decade later, the Centers for Disease Control (Segal, 1994) provided print based correspondence study in public health topics. When technology permitted, they adopted telephone, compressed video and satellites to enhance instruction. 
In a primer for distance education in public health, Segal (1994) stated that, "Although print based instruction can allow learners to apply and assess their knowledge in simple ways, used alone, it does not easily allow the practice and assessment of complex skills. Computers provide an excellent means to engage individual learners in active problem solving in a realistic way."

The passage of several decades has made most instructional material available on television outmoded. Improvements in technology have included cost reduction, high definition signals in the 1990s and digitisation in the 2000s. The advent of digital television is significant. Software of an educational nature can now be packaged with DVDs and the interactive TV that digital technology promises will increase programming and training options.

\section{Multimedia capacity}

A crucial step for multimedia and the development of attractive educational software was taken in 1985 when Windows 1.01 was introduced with Aldus PageMaker, a desktop publishing program. With the release of Windows 2.01 in 1987, a fifth of the US States were using the new technology for some form of education.

In the mid-nineties Windows 95 and Windows NT offered serious multimedia platforms with 32-bit addressing. Other operating systems preceded them in this but did not achieve their massive distribution. The new versions of Windows allowed multiple programs to run simultaneously and gave each program a virtual space of 2 gigabytes of RAM. Physical memory also grew swiftly (personal computers now routinely have $256 \mathrm{MB}$ of RAM and can have much more). This was a critical step because multimedia programs are very heavy users of addressable RAM space. It goes without saying that simulations cannot be lifelike unless they are capable of supporting graphic components. But this was only possible with parallel advances in hard disk capacity. Movie files were simply too big for older computers to handle and a minimum of 20 GB of hard disk space is currently recommended for movie making on personal computers. Size was not the only problem. Hard disks running at $3,000 \mathrm{rpm}$ were too slow to carry the high rate of information required to portray a movie without jerky movement. Speeds have increased in the past few years and hard disk drives that run at 7000-10000 rpm and support smooth motion are now widely available.

\section{Educational software development}

The above mentioned technological advances promoted computers into the forefront of the technologies that support education. Even in 1980, over 500 
educational software programs were available for use on Apple, TRS-80 and PET microcomputers (Dresden Associates 1980). The number of programs in circulation in 2001 is not possible to measure.

To get a picture of trends in educational software activity, a search was made in the Cambridge Scientific Abstracts Internet Search Database (CSA 2001) of the term "educational software" (Figure 1). The publication pattern over the decades, indicating a sharp increase and then a decline in activity, was almost identical to that obtained by Buckleitner (1999), who surveyed the ERIC database for the terms "Software and Evaluation."

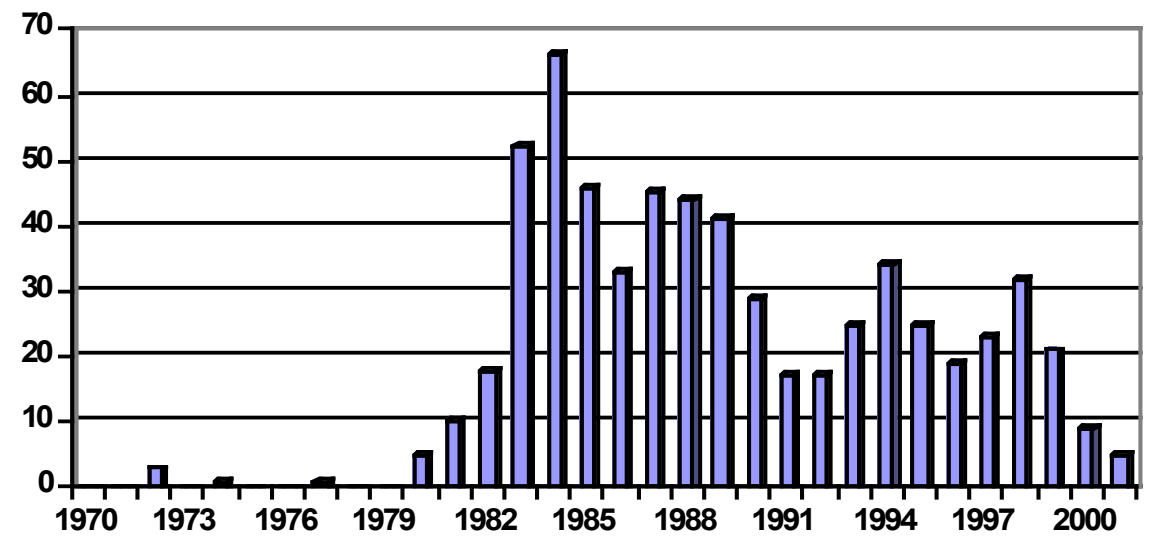

Figure 1: Number of publications listed in the CSA Internet Database Service (CSA 2001) when the search term "Educational Software" was entered.

The very recent advances in hardware and software described above led to an impressive explosion of computer capability that is still well beyond current developments in educational software applications. Note the small bounce back in publications after widespread Web access was achieved in 1995. This slow progress is possibly accounted for by the fact that the various multimedia elements still had to be drawn together into integrated software products. Macromedia Flash is a recent example since it assimilates most multimedia types in a single online presentation. This combining of different media under program control, so necessary for the development of educational software, has taken the five years between the emergence of the 32-bit environment and the present. Another example is that the primary software medium of the web, HTML, has only recently become fully integratable within other software packages. 
Much educational software is only available on CD due to the large size of multimedia files and the inability of web based applications to provide solutions with adequate speed. The availability of wide bandwidth will mean that some applications now on $\mathrm{CD}$ will soon be available directly from online servers. The IDC (2000) stated that the number of broadband users in Australia is forecast to reach 4.5 million by 2005 . Nevertheless, software with extensive multimedia components will still run more efficiently on stand alone computers. A more flexible combination solution, in which the main application runs on a stand alone computer, but is supplemented by integrated web access, is likely to be widely used. Some software products running stand alone can already update themselves from the web, and the libraries they use can already be kept current by automatically synchronising with web based resources. Such 'best of both worlds' solutions have an obvious relevance for educational software.

\section{The Internet and the World Wide Web}

In 1995, the US Federal Networking Council defined the term Internet. "Internet refers to the global information system that -- (i) is logically linked together by a globally unique address space based on the Internet Protocol (IP) or its subsequent extensions/follow ons; (ii) is able to support communications using the Transmission Control Protocol/Internet Protocol (TCP/IP) suite or its subsequent extensions/follow ons, and/or other IP-compatible protocols; and (iii) provides, uses or makes accessible, either publicly or privately, high level services layered on the communications and related infrastructure described herein" (DARPA 2001).

The Internet is thus the software and hardware supporting the abstract space of networks we now know as the World Wide Web or just 'the Web'. It was designed before Local Area Networks (LANs) existed, and readily accommodated to that technology in addition to absorbing electronic mail and desktop publishing.

Recent developments in hardware and software provide powerful resources for the development of advanced multimedia applications and presentations. The only limiting factors are new ideas on how to use these technological advances for educational purposes. We now turn to educational theory and some of its contribution to date to the development of these enabling ideas.

\section{Public health software for education}

Software specifically produced for public health education is very limited. An attempt to quantify the extent of software development applicable to 
public health education was made by searching for the terms 'public health', 'software' 'education', 'health', and 'training' in the Google and Yahoo! search engines, in major public health sites and associations and in software search engines. These searches revealed many applications that focussed on epidemiology, data analysis and management, health administration, surveillance, and medical or hospital education and administration. A variety of other health related programs were found on dietary management, fitness, behaviour monitoring, nutrition, personal medical records, genealogy and mood modification.

Although some of this software could train public health students in these, most was not produced with education in mind. As well, the software could in the main be described as highly specific and hard coded to address very particular applications.

A developer of 21st century educational software can learn something from educational theories such as anchored instruction (CTGV 1997), case based learning (Jonassen, 1999), cognitive flexibility learning (Spiro \& Jehng, 1990), goal based scenario learning (Schank, 1995), open ended learning (Hannafin et al, 1994), constructivist learning (Jonassen, 1999) and problem based learning (Savery \& Duffy, 1994; Grabinger, 1996). What unites most if not all of these approaches is an underlying idea: Learning will be most effective when realistic problems are presented that call forth a creative effort when a student attempts to solve them.

A literature search using PubMed revealed 500 plus responses from 1995 to the present. Very few actual software applications that could be used in public health education other than epidemiology or statistics were found. Of note were applications evaluated and/or developed such as an evaluation of an infection control training package (Desai et al. 2000), a computer based radiation safety training program (Hamilton et al. 2000), a automated external defibrillation skill builder for emergency medical technicians (Jerin et al. 1998) and Do Epi, an epidemiology program (Dean et al. 1998). Do Epi is notable because it has the ability to construct new exercises, which could include hypertext and low resolution graphics. One of this program's greatest negative points was expressed by the authors. "DoEpi is based on DOS programs to allow the widest use. The format lends itself to conversion to hypertext programs in Microsoft Windows and Internet formats at a future date." Unfortunately DOS based programs are no longer acceptable to most students and this statement truly highlights the backward condition of educational software in public health.

The PEP registry for parents, educators and publishers currently lists an incredible 2207 publishers that are producing educational software (PEP 2001). This is clear evidence that educational software is a booming 
business. It is thus hard to comprehend why there are so few educational software applications that have been written for public health.

\section{Online and distance learning}

Compact disc based and online learning is proliferating to the extent that most universities offer some form of distance education and support to their students. Web usage has increased dramatically with the number of adult users in Australia rising to 6.9 million or nearly $50 \%$ of the population in 2000 (ABS 2001). The IDC (2000) estimated the number of online users worldwide at 513.4 million.

Among the problems that have faced the development of attractive and accessible teaching materials have been steep learning curves, time constraints and data speeds over standard telephone lines. The latter issue acts to restrict the amount of data that can be transmitted in a reasonable amount of time, thus limiting the size of Web pages and the use of images. This can be a major issue for students in rural or remote areas who wish to access online learning. Despite the availability of the Web and its geometric rate of expansion and provision of resources, the print medium, constituting little more than the traditional correspondence course, remains today the dominant form of distance education.

In recent years, there has been a proliferation of online courses and subjects, but most are still print based. They mimic regular textbooks, having a linear structure and being arranged into a series of Web pages that follow on from each other. Help screens are an example of this structure. Although there are hypertext links between pages, allowing the user greater control, there is no interaction between the information and the learner. Video transmissions of lectures have also become widely available on the Web. Similarly, sound has been added to PowerPoint slides so that a learner can hear a lecture on the web along with presented images. Just like text based presentations, these transmissions are not interactive.

The simplest or linearly interactive material comes from structured tutorials or programmed instruction, which previously existed in book form. Tutorials most commonly present information in small pieces to carefully explain a theory or process. Some provide this experience via detailed help screens attached to general text. Interspersed amongst the instructional material there are scenarios or problems followed by a simple question. The student's answer to the question is then evaluated and basic feedback that may or may not include additional information is provided (Alessi and Trollip, 1985). 
It is understandable that educators made full use of existing methods and were slow to develop entirely new forms of interaction. The key is the required effort. Interactive programs take time and effort to write. Further, the availability of user friendly web development software is a very recent phenomenon and educational providers are still to make widespread use of it (Idaho College of Engineering, 1995).

In 1992, the Pennsylvania University Task Force on Distance Education stated that "the need for new models of programs, assessment, management and marketing specifically developed for distance education is already great, but no one has, as yet, come forward to design and create them" (Task Force on Distance Education 1992). Although matters have advanced somewhat over the past decade, little use has been made of hitech approaches to learning and most online distance education materials are still very similar to printed materials. There is thus a clear need for the development of improved andragogy or adult based, student centered, self directed learning environments (Pellone 1995) which can improve motivation, performance, retention and deliver a deeper and more profound educational experience.

\section{Online and distance public health solutions}

Online flexible education strategies make a lot of sense in training the public health workforce. The workforce consists of mid-career health professionals who are, for the most part, employed and geographically dispersed. Physical attendance is thus expensive in terms of travel, lodging costs and time lost from work. Short courses designed to impart considerable information in 2-4 days can in practice be less cost effective even though they conserve time. Flexible options are attractive because they allow a learner to proceed at their own pace at a convenient time. The provision of materials in a number of formats can enable the learner to read, view or interact when they wish without interrupting their other responsibilities. Most health professionals are already adept in certain fields. Flexible learning allows them to revisit, at their convenience, what they have difficulty with and to skim over what they already know.

Examples of possible candidates for providing more effective educational solutions are decision support systems, modelling and simulation software. As we shall see later, these three approaches developed separately but can now be combined to provide a more comprehensive approach.

\section{Decision support systems}

The idea of creating a system that could facilitate the decision making process originated from theoretical investigations into organisational 
decision making and interactive computer research before the advent of the personal computer (Keen and Morton 1978). Various aspects of the concept were explored in the 1970s (Little 1970; Gerrity 1971; Morton 1971; Keen and Morton 1978; Rockart 1979), however, the focus was almost entirely on executive management decision making and support applications.

Decision support systems (DSS) are well suited to public health applications due to the multidisciplinary and investigative nature of many public health tasks. Conventional training aims to provide a knowledge base and examples of how to implement the knowledge in resolving issues of public health concern, be they related to health informatics (Nykanen, 2001), disease management (Sanson et al, 1999; Johnson et al, 2000; Canyon, 2001), environmental health management (Schreider et al, 1999; McLaughlin and Kirkpatrick, 2000; Schreider and Mostovaia, 2001) or patient diagnosis and management (Scott and Lenert, 2000; Abidi, 2001; Ruland, 2001; Weiner and Pifer, 2001) and evidence based clinical medicine (Sim et al, 2001). As can be noted from these topics, decision support systems are a typical example of the goal based learning approach.

\section{Models}

Very many mathematical models that have been written to shed light on multitudes of physical and health situations. Benz (undated) provided an exhaustive list of ecological models and those that relate to environmental health, such as climate change, toxicological, pesticide breakdown, bioaccumulation of pollutants and pollutant movement models, are somewhat relevant to public health. More relevant are the less abundant disease propagation models. These models accept certain input from the user, some of which relate to population, host, vector, immunity, and infection parameters. They then run for a defined period or until an epidemic has exhausted itself. In this way, the effect of variations on management or control efforts can be predicted and decisions can be made. Models are an example of the open ended learning approach. When mathematical models become integrated with the ability to control model variables, advanced simulations can be created.

\section{Simulations}

Simulations represent the most sophisticated form of interaction available between the learning environment and the learner, because they can potentially include aspects of DSS and modelling to create more realistic scenarios. Users are expected to make process or pathway decisions in a problem based "role playing situation" (Lillie et al 1989). "The strength of a simulation is the fact that a computer responds to student input. That is, 
the computer's responses depend on the choices students make" (Geisert and Futrell 1990). Responses may be predetermined if the pathway is expected or alternatively, may be calculated in real time.

Problem solving, as an educational method, is eliciting much interest and many have argued that problem solving should form a major part of education. Problem solving simulation software has been defined as programs that teach the steps involved in solving problems directly through explanation and/or practice, or programs that help learners acquire problem solving skills by giving them the opportunities to solve problems (Roblyer et al, 1997). Each student response brings about a change in the problem state, with the new state being offered to the student for another response. Students proceed through many levels of interaction as they progress deeper into the model in an attempt to solve a particular problem.

The computational procedures can be embedded in user friendly interfaces written in a RAD (rapid application development) environment. The concept is to convert outputs from the computed equations into descriptions of changes in the problem situation, so that lifelike scenario changes are presented to the student as a reaction to his or her efforts. The output descriptions are primarily textual, but appropriate supplementation using graphs, sound (vocal response), movie clips and other visuals is possible.

Simulation methods can be divided into a further four categories (Alessi and Trollip, 1985): physical, procedural, situational and process.

Physical simulations provide a physical environment such as an automobile dashboard, airplane cockpit or entire model space shuttle and are primarily created because of the cost or risk involved in learning on the "real thing". Procedural simulations guide the learner through lists of actions that may be required to fix something complex such as airplane wiring. Situational simulations present the learner with scenarios and rely on decision based input from the learner to change the problem or situational state. Process simulations are those that allow the learner to experiment with various influencing parameters in a protected environment.

The last class of simulation type software concerns educational computer games. If well written, they can be motivating and the learner can acquire important problem solving skills. The major difference with this type of software is the element of competition either at a student, group or computer level. The development of simulation modelling technologies will result in the creation of several novel market areas for training applications based on the simulation of complicated systems. 
- Higher education sectors are viable markets due to their large client base. Educational software is increasingly adaptive to student needs and should be robust enough to make the transition to the online environment.

- Simulations are increasingly used in environmental areas where they model precautionary or investigative activities such as investigation of chemical spills.

- Prognostic and diagnostic simulations in medical areas can be used to train personnel in the operation of expensive and/or complex instrumentation or difficult surgical techniques.

- In the area of infectious disease control, simulation models such as ONCHOSIM (Plasier et al, 1990) have been developed for analysis, evaluation and prediction. The Onchocerciasis Control Program in West Africa has used ONCHOSIM over the past decade and recommends it as a practical tool.

\section{Next generation simulators}

When the categories of simulation methods are assessed, it becomes clear that next generation simulators will include aspects of the physical category, because they can portray before and after effects of user decisions in a graphical context. The procedural category will also be included because users can be guided through a simulation by a set sequence of logical questions and decisions. The situational category can be combined with the others because decisions can be made within a scenario based application. Lastly, the process category can be included because users can return to alter past decisions and experiment with various influencing parameters in a protected environment.

One of the advantages of integrated simulations is that they cater for different learning philosophies. Providing somewhat randomly organised materials from different viewpoints fosters the development of research skills. The user is required to bring order and synthesis to the materials in order to present evidence to support an argument or make a decision. Training the user in the application of material sequencing can also foster flexible analytical skills. Creating an environment with a suitable structure and appropriate sequencing of multimedia in which the user can navigate without confusion can greatly increase the effectiveness of the knowledge being communicated (Bates and Ellis 1995).

Integrated simulations are most closely associated with cognitive flexibility, where the learner is expected to evaluate and assimilate information from multiple origins and apply it to solve a realistic case based problem, and 
with goal based learning, where tasks can be based on design, diagnosis, discovery or control.

\section{Designing a training simulator}

One of the problems with most mathematical models and simulations is that they are aimed at highly capable end users and their user interface is hard coded with little built in flexibility. This, coupled with their singularly complex level of operation, usually means that they are unsuitable for training purposes. Training simulations must by their very nature have at least two interfaces: a developer interface and a trainee interface. For example, business management simulations have a supervisor level in which the rules of operation are defined and a player interface in which decisions can be made within a defined framework. But even these interfaces are highly specific and are hard coded without much flexibility.

A more broad based solution would be to have a general purpose simulation model writer that could provide the required flexibility and power to generate a variety of decision support simulations. The integration of multimedia with a simulator is not without difficulties. From a programming perspective, connecting simulation elements to specific media and online content is a novel idea. As a first condition, the simulation must be stepwise in nature rather than being generated from differential equations in a single step, as in an epidemiological model. The ability to script code within the simulator is another obvious design element. There must be a language of sorts to connect the underlying decision support system to estimates of risk, project outcomes and evaluation, and multimedia content. This implies that the software must be general purpose in design, enabling comprehensive flexibility in the creation of the simulation. Stating the need in one sentence does not make it any easier to accomplish. Software designers will let it be known that designing a general purpose user interface is fraught with programming complexities.

\section{Educational evaluation}

The design of a training simulator would be incomplete if it did not include some aspect of self assessment. The design outlined above is complete in that it not only can be used to guide and limit the movement of a learner through a learning environment, but it can also assess the final learning outcome. The evaluator would be able to assess the pattern of choices made by the learner, the resources that were accessed and the final condition of project outcome factors. There is less restriction in this design than that imposed by drills, which take the learner stepwise through a series of problems and restrict learner progress until a satisfactory answer is 
obtained. Drills cater for different learning paces and enable speedy progression for those who are sufficiently capable. They are limited, however, because they prevent a learner from seeing the future implications of inappropriate decisions.

A major issue of concern in online evaluation is that of ensuring learner identity. If there is no way of guaranteeing the identity of the person who has taken a test or has produced an assignment, the whole system collapses.

As stated in all the learning approaches, simulations must be interesting and motivating. For the distance learner, who is beyond the influence of charismatic lecturers, software solutions involving multimedia and problem solving must stand at the apex of the motivation pyramid.

\section{Next generation educators}

With the whole educational system in such a state of flux and change, it is becoming difficult for educators to know which areas of educational technology to emphasise and how to emphasise them. We are witnessing the gradual emergence of globally accepted teaching materials, such as textbooks and manuals, so will the next step be nationally or internationally accepted, automatically updated curriculum and competency standards? The trend towards online learning can only be welcomed by teaching institutions who will not have to provide as much infrastructure and who may look forward to better results from their students.

In the online world, what will be the role of future educators with regard to online learners? The shift in mode of information delivery, when stabilised and running smoothly, will release academics from many of the traditional burdens within the educational process, enabling them to research and design new modes of training that go beyond information delivery. Once the technological hurdles are surmounted, academics may well be grateful for additional time to conduct research and produce publications and feed the results back into their training environments. On the whole, the situation looks quite promising.

\section{References}

Abidi, S. S. (2001). Knowledge management in healthcare: Towards 'knowledgedriven' decision support services. International Journal of Medical Informatics, 63, $5-18$.

ABS (2001). Use of the Internet by adult householders: November 2000. Australian Bureau of Statistics 8147.0. [viewed 20 Nov 2001, verified 2 May 2002] http: / / www.abs.gov.au / Ausstats / abs@.nsf/Lookup/ NT00010206 
Alessi, S. M. \& Trollip, S. R. (1985). Computer-based instruction: Methods and development. Prentice-Hall, New Jersey.

An, J. S. (2001). The early years: 1913-1940. Instructional systems technology. School of Education. Indiana University, Bloomington. [viewed 20 Nov 2001, verified 2 May 2002] http: / / www.indiana.edu/ ist/students / history / early.html\#para3

ANAPHI (2001). Australian Network of Academic Public Health Institutions. [viewed 20 Nov 2001, verified 2 May 2002] http: / / www.anaphi.unsw.edu.au/index.htm

Anon (2001). Founding Dean Elizabeth Soule was a public health pioneer. University of Washington, Seattle, Washington, USA. [viewed 20 Nov 2001] http:/ / www.son.washington.edu/about/elizabeth-soule.asp [verified 2 May 2002 at http:/ / www.son.washington.edu/connections/win99/legacies_of_leadership.asp]

Bates, T. \& Ellis, M. (1995). Who's in charge here? Managing multimedia projects. Campus Computing and Communications, University of British Columbia. [viewed 20 Nov 2001, verified 2 May 2002] http:/ / www.cc.ubc.ca/ campuscomputing/sep95/multimedia.html

Benz, J. (undated). Register for ecological models. WWW-Server for Ecological Modelling, European Chapter. International Society for Ecological Modelling. [viewed 20 Nov 2001, verified 2 May 2002] http:/ / dino.wiz.unikassel.de/ecobas.html

Bergman, M. K. (2000). The Deep Web: Surfacing hidden value. BrightPlanet.com LLC. [viewed 20 Nov 2001, verified 2 May 2002]

http: / / www.completeplanet.com/tutorials / deepweb/index.asp

Buckleitner, M. (1999). The state of children's software evaluation. Yesterday, today and in the 21st century. Childrenssoftware.com [viewed 20 Nov 2001, verified 2 May 2002] http: / / www.childrenssoftware.com/ evaluation.html

Canyon, D. V. (2001). Web-based distance education and the future of simulation models. In D. V. Canyon, S. McGinty \& D. Dixon (Eds), Tertiary teaching IV: Flexible teaching and learning across the disciplines. Craftsmen Products Online Publications, Sydney. [viewed 20 Nov 2001, verified 2 May 2002] http: / / www.craftsmenproducts.com/gctt01/

CSA (2001). Cambridge Scientific Abstracts. Internet Database Service. [viewed 20 Nov 2001, verified 2 May 2002] http: / / www.csa3.com/csa/index.html

CTGV - Cognition \& Technology Group at Vanderbilt (1997). The Jasper Project. Mahwah, NJ: Lawrence Erlbaum Associates.

DARPA (2001). Mission and Background. Defense Advanced Research Projects Agency. [viewed 20 Nov 2001] http:/ / www.arpa.mil/

Dean, A. G., Shah, S. P. \& Churchill, J. E. (1998). DoEpi. Computer-assisted instruction in epidemiology and computing and a framework for creating new exercises. American Journal of Preventative Medicine, 14, 367-371. 
Desai, N., Philpott-Howard, J., Wade, J. \& Casewell, M. (2000). Infection control training: evaluation of a computer-assisted learning package. Journal of Hospital Infections, 44, 193-199.

Dresden Associates (1980). School microware: A directory of educational software. Over 500 programs for Apple, PET, TRS-80. Dresden, Maine.

Geisert, P. \& Futrell, M. (1990). Teachers, computers, and curriculum: microcomputers in the classroom. Allyn and Bacon, Needham Heights MA.

Gerrity, T. P. Jr. (1971). The design of man-machine decision systems. Sloan Management Review, 12, 59-75.

Grabinger, R. (1996). Rich environments for active learning. In D. H. Jonassen (Ed), Handbook of Resarch for Educational Communications and Technology. Macmillan, New York.

Hamilton, D. S., Peck, M. M., Yu, H. \& Kearfott, K. J. (2000). Computer-based radiation safety training for hospital radiation workers. Health Physiology, 78, S48.

Hannafin, M. J., Hall, C., Land, S. M., \& Hill, J. (1994). Learning in open environments: Assumptions, methods and implications. Educational Technology, $34,48-55$.

Idaho College of Engineering (1995). Distance education at a glance. University of Idaho. [viewed 20 Nov 2001, verified 2 May 2002] http: / / www.uidaho.edu/ evo/dist1.html

IDC (2000). Analyse the future: Australia. [viewed 20 Nov 2001, verified 2 May 2002] http:/ / www.idc.com.au /

Jerin, J. M., Ansell, B. A., Larsen, M. P. \& Cummins, R. O. (1998). Automated external defibrillators: skill maintenance using computer-assisted learning. Academy of Emergency Medicine, 5, 709-17.

Johnson, P. D., Tu, S., Booth, N., Sugden, B. \& Purves, I. N. (2000). Using scenarios in chronic disease management guidelines for primary care. Proceedings of the AMIA Symposium, 389-393.

Jonassen, D. H. (1999). Designing constructivist learning environments. In C. M. Reigeluth (Ed), Instructional Design Theories and Models, Vol. II: A New paradigm of instructional theory. Mahwah, NJ: Lawrence Erlbaum Associates.

Keen, P. G. W. \& Morton, M. S. S. (1978). Decision Support Systems: An Organizational Perspective. Addison-Wesley, Inc. Reading, MA.

Lillie, D. L., Hannum, W. H. \& Stuck, G. B. (1989). Computers and effective instruction: using computers and software in the classroom. Longman Publishing Group, New York.

Little, J. D. C. (1970). Models and managers: The concept of a decision calculus. Management Science, 16, B466-485. 
Morton, M. S. S. (1971). Management decision systems; computer-based support for decision making. Division of Research, Graduate School of Business Administration, Harvard University, Boston.

Myatt, M. (2001). Some Free Public Health Software. [viewed 20 Nov 2001] http: / / www.myatt.demon.co.uk/

Nykanen, P. (2001). On conceptualization of a decision support system in health informatics. Medinfo 10, 503-507.

Pellone, G. (1995). Educational software design: a literature review. Australian Journal of Educational Technology, 11, 68-84. [viewed 20 Nov 2001, verified 2 May 2002] http:/ / www.ascilite.org.au/ajet/ajet11/ pellone.html

PEP (2001). PEP registry of Educational Software Publishers. PEP: Resources for Parents, Educators and Publishers. [viewed 20 Nov 2001, verified 2 May 2002] http:/ / www.microweb.com/pepsite/Software/publishers.html

Plasier, A. P., Van Oortmarssen, G. J., Habbema, J. D. F., Remme, J. \& Alley, E. S. (1990). ONCHOSIM: a model and computer simulation program for the transmission and control of onchocerciasis. Computer Methods and Programs in Biomedicine, 31, 43-56.

Roblyer, M. D., Edwards, J. \& Havriluk, M. A. (1997) Integrating educational technology into teaching. Merrill, Upper Saddle River, New Jersey.

Rockart, J. F. (1979). Chief executives define their own data needs. Harvard Business Review, 67, 81-93.

Ruland, C. M. (2001). Developing a decision support system to meet nurse manager's information needs for effective resource management. Computing and Nursing, 19, 187-193.

Sanson, R. L., Morris, R. S. \& Stern, M. W. (1999). EpiMAN-FMD: a decision support system for managing epidemics of vesicular disease. Review of Science Technology, 18, 593-605.

Savery, J. R., \& Duffy, T. J. (1994). Problem based learning: An instructional model and its constructivist framework. Educational Technology, 35, 31-38.

Schank, R. C. (1995). Engines for Education. Hillsdale, New Jersey: Lawrence Erlbaum Associates.

Schreider, S. Y. \& Mostovaia, A. D. (2001). Model sustainability in DSS design and scenario formulation: What are the right scenarios? Environment International, 27, $1-6$.

Schreider, S. Y., Scoccimarro, M., Jakeman, A. J., Dietrich, C. R., Saowapon, S. \& Merritt, S. (1999). Hydrological components of a DSS for sustainable developments of northern Thailand highland catchments. 2nd International Conference on Multiple Objective Decision Support Systems (MODSS99), Brisbane.

Scott, G. C. \& Lenert, L. A. (2000). What is the next step in patient decision support? Proceedings of the AMIA Symposium, 784-788. 
Segal, B. S. (1994). Distance learning: The instructional strategy for the 90s. Division of Media and Training Services, Public Health Practice Program Office, Centers for Disease Control and Prevention. [viewed 20 Nov 2001] http: / / cdc.gov/phtn/ primer.htm

Sim, I., Gorman, P., Greenes, R. A., Haynes, R. B., Kaplan, B., Lehmann, H. \& Tang, P. C. (2001). Clinical decision support systems for the practice of evidence-based medicine. Journal of the American Medical Informatics Association, 8, 527-534.

Spiro, R. J., \& Jehng, J. (1990). Cognitive flexibility and hypertext: Theory and technology for the non-linear and multidimensional traversal of complex subject matter. In D. Nix \& R. J. Spiro (Eds), Cognition, Education and Multimedia. Hillsdale, NJ: Lawrence Erlbaum Associates.

Task Force on Distance Education (1992). Report of the Task Force on Distance Education. Pennsylvania State University. [viewed 20 Nov 2001, verified 2 May 2002] http:/ / www.ed.psu.edu/acsde/deos/deosnews / deosnews3_7.asp

Weiner, M. G. \& Pifer, E. (2000). Computerized decision support and the quality of care. Management Care, 9, 41-51.

Zenger, J. T. \& Walker, T. J. (1999). Impact of the Internet on entomology teaching and research. [viewed 20 Nov 2001]

http: / / csssrvr.entnem.ufl.edu/ walker / fewww / ZengerARE.htm [verified 2 May 2002 at http:/ / csssrvr.entnem.ufl.edu/ walker/epub/ZengerARE.htm]

Dr Deon V. Canyon, School of Public Health and Tropical Medicine, James Cook University, Townsville, Queensland 4811, Australia Email: Deon.Canyon@jcu.edu.au

David N. Podger, Craftsmen Products Pty Ltd 